\title{
LMOOC para la integración de personas desplazadas
}

\section{LMOOCs to integration of displaced people}

\author{
Mercedes SANZ GiL \\ Universitat Jaume I \\ sanzg@uji.es
}

\begin{abstract}
Résumé
L'un des principaux défis auxquels les personnes déplacées sont confrontées est celui de faire face aux barrières linguistiques et culturelles qui découlent du manque de connaissance, dans la plupart des cas, de la langue et de la culture du pays de destination. De nos jours, et grâce aux Technologies de l'Information et de la Communication (TIC), il existe de nombreuses ressources et applications qui peuvent contribuer à pallier ces lacunes, avant, ou pendant la période de mobilité. Dans cet article, nous en présentons quelquesunes. Nous nous concentrons en particulier sur le format MOOC (Massive Open Online Courses) pour l'apprentissage des langues étrangères (LMOOC). Nous analyserons deux LMOOC pour l'apprentissage de l'Espagnol comme Langue Étrangère (ELE) destinés principalement à des personnes migrantes et réfugiées et trois pour l'apprentissage du Français comme Langue Étrangère (FLE) destinés principalement aux personnes en déplacement pour des raisons professionnelles ou éducatives.
\end{abstract}

\section{Mots-clé}

Espagnol Langue Étrangère, Français Langue Étrangère, MOOC, personnes déplacées, TIC.

\begin{abstract}
One of the main challenges faced by displaced people is dealing with the linguistic and cultural barriers that arise from the lack of knowledge, in most cases, of the language and culture of the destination country. At present, thanks to Information and Communication Technologies (ICTs), there are numerous resources and applications that can help to alleviate these deficiencies, before, or during the period in which mobility develops. In this article we present some of them. More specifically, this article focuses on the MOOC (Massive Open Online Courses) format for foreign language learning (LMOOC). We will analyze two LMOOCs for learning Spanish as a Foreign Language (SFL) aimed mainly at migrants and refugees; and three for learning French as a Foreign Language (FFL) aimed mainly at people displaced for work or educational reasons.
\end{abstract}

\section{Key-words}

Spanish as a Foreign Language, French as a Foreign Language, MOOCs, displaced people, ICTs. 
Anales de Filología Francesa, n. ${ }^{\circ}$ 29, 2021

LMOOC PARA LA INTEGRACIÓN DE PERSONAS DESPLAZADAS

\section{Introducción}

La Oficina del Alto Comisionado de las Naciones Unidas para los Refugiados (ACNUR), en su informe anual sobre tendencias globales de desplazamiento forzado, registraba a final de 2019 un dato que batía el récord de los últimos 10 años: 79'5 millones de personas en el mundo se encontraron en situación de desplazadas como resultado de la persecución, el conflicto, la violencia, las violaciones de los derechos humanos u otros eventos que alteraron gravemente el orden público (ACNUR, 2020).

Si a esta cifra sumamos la de las personas desplazadas por motivos profesionales, como desplazamiento temporal promovido por la empresa, o como desplazamiento a mediolargo plazo en busca de un empleo, el número aumenta exponencialmente. Como referencia orientativa de una fecha cercana, en 2017, la Organización Internacional del Trabajo registraba 168 millones de migrantes laborales (ILOSTAT, 2018).

Otro colectivo de personas desplazadas que nos interesa mencionar en este trabajo es el estudiantado universitario que viaja temporalmente a otros países para realizar una parte de sus estudios. A modo de ejemplo mencionamos el bien conocido programa de movilidad estudiantil Erasmus. En su informe anual de 2019 registraba 940.000 personas que habían realizado una movilidad de estudios o prácticas formativas (Comisión Europea, 2020).

Uno de los principales retos a los que deben enfrentarse todas ellas, sea cual sea el motivo de su desplazamiento, es hacer frente también a las barreras lingüísticas y culturales que supone el desconocimiento, en la mayoría de los casos, de la lengua, de la cultura, de las costumbres y de la cotidianeidad del día a día del país que les acoge (Sanz, 2020).

En pleno siglo XXI y teniendo como telón de fondo los Objetivos de Desarrollo Sostenible ${ }^{1}$ definidos en 2015 por la Organización de las Naciones Unidas como hoja de ruta para los siguientes 15 años (Agenda 2030), desde los sectores educativos tenemos la responsabilidad y la obligación moral de investigar en propuestas que contribuyan a derribar estos obstáculos mediante formación lingüística e intercultural.

Actualmente, y gracias a las Tecnologías de la Información y la Comunicación, existen numerosos recursos y aplicaciones que pueden ayudar a paliar dichas carencias, antes, o durante el periodo en el que se desarrolla la movilidad. En este artículo presentamos algunos de ellos. En concreto nos centramos en el formato MOOC (Cursos masivos abiertos en línea, por sus siglas en inglés de Massive Open Online Courses) para el aprendizaje de lenguas extranjeras (LMOOC), Language MOOC.

1 Los 17 Objetivos de Desarrollo sostenible identificados por la ONU son: Fin de la pobreza; Hambre cero; Salud y bienestar; Educación de calidad; Igualdad de género; Agua limpia y saneamiento; Energía asequible y no contaminante; Trabajo decente y crecimiento económico; Industria, innovación e infraestructura; Reducción de las desigualdades; Ciudades y comunidades sostenibles; Producción y consumo responsables; Acción por el clima; Vida submarina; Vida de ecosistemas terrestres; Paz, justicia e instituciones solidarias; Alianzas para lograr los objetivos (https://www.un.org/sustainabledevelopment/es/sustainable-development-goals/) [31/05/2021] . 
El formato MOOC, concepto formativo creado por Georges Siemens y Stephen Downes en 2008 (Pernías \& Luján, 2013), se creó con la finalidad de favorecer la democratización de la formación y del conocimiento, facilitando el acceso a un vasto público, pues no suelen tener límite de matrícula. Además, por su carácter gratuito y exigencias tecnológicas mínimas, ya que solo se requiere una simple conexión a Internet y un dispositivo electrónico que la permita, se revela como un entorno idóneo para contribuir con la educación de calidad, para favorecer el crecimiento económico o reducir las desigualdades entre personas, respondiendo así a algunos de los Objetivos de Desarrollo Sostenible mencionados, pues, se trata de entornos de aprendizaje abierto que, desde la concepción conectivista (Siemens, 2004) que los caracteriza, suponen un punto de encuentro de miles de personas procedentes de cualquier rincón del planeta accediendo a numerosas propuestas educativas, de temáticas variadas, ofrecidas por instituciones de reconocido prestigio.

Desde que surgió el fenómeno MOOC ha habido un extenso debate en cuanto a la tipología (Sedano, 2017) identificando numerosos modelos de MOOC en función de objetivo. Por mencionar algunos, los MOOC conectivistas (cMOOC), los sociales (sMOOC), de corte pedagógica más tradicional (xMOOC), centrados en tareas (tMOOC), en juegos (gMOOC) o para el aprendizaje de segundas lenguas (LMOOC), entre otros.

Nos interesa para este trabajo el último modelo mencionado. Según Bárcena \& Martín-Monje, autoras que acuñaron el término en 2014, los objetivos de los Language MOOC, además de la educación formal regular, pueden abarcar la construcción de bases para estudiantes de todo tipo de niveles; la formación a medida para personas que necesitan actualizar capacidades específicas en una segunda lengua; y también la formación no formal para quienes están fuera de la educación formal e incluso en situaciones vulnerables de exclusión profesional y social. A este respecto, los LMOOC podrían no sólo ayudar a estos alumnos a satisfacer sus necesidades lingüísticas, sino también tender puentes hacia la educación formal (Bárcena \& Martín-Monje, 2014).

Esta peculiaridad masiva y conectivista pone en valor el encuentro entre personas de diferentes procedencias y culturas, propiciando de manera natural la formación intercultural que toda propuesta de aprendizaje de lenguas requiere. Según Mathilde Anquetil (2006), el objetivo de la formación intercultural es favorecer el encuentro entre personas de diferentes orígenes y procedencias. Dicha formación puede realizarse "mejorando las condiciones de receptividad por medio de la enseñanza de diferentes hechos culturales o diseñando actividades que obliguen al aprendiz a interactuar con el medio, actividades para adquirir conocimientos cuya finalidad sea crear situaciones de encuentro" (Anquetil, 2006: 312).

En este artículo describiremos varios LMOOC de aprendizaje de lenguas extranjeras para facilitar la integración lingüística y cultural de los tres colectivos de personas desplazadas que hemos mencionado anteriormente: dos LMMOC aprendizaje del Español como Lengua Extranjera (ELE) destinados principalmente al colectivo de personas mi- 
grantes y refugiadas y tres LMOOC de aprendizaje del Francés como Lengua Extranjera (FLE) destinados principalmente a personas desplazadas por motivos laborales o educativos.

Estos LMOOC han sido seleccionados entre una oferta de 37 disponibles durante los meses de abril y mayo de 2021 que se ajustaban a los siguientes criterios de búsqueda: (1) lengua de instrucción: francés o español, (2) objeto de aprendizaje: la lengua francesa o la lengua española bien como lenguas maternas, o como lenguas extranjeras o segundas, (3) periodo de realización de los cursos: abril y mayo de 2021 o de acceso libre en cualquier momento.

La mayoría de ellos están alojados en las plataformas especializadas en formación MOOC Coursera, OPENedX o Miriadax. Sobre cada uno de los 5 LMOOC describiremos la autoría y entorno tecnológico que los aloja, los objetivos, las principales características técnicas, lingüísticas y pedagógicas y los contenidos tratados. Por último, mencionaremos aquellos aspectos que especialmente favorecen el encuentro intercultural y, por ende, facilitan la integración de las personas desplazadas en la sociedad de acogida.

\section{LMOOC para el aprendizaje del Español como Lengua Extranjera}

Los LMOOC para el aprendizaje del ELE que presentamos a continuación llevan por título: Puertas Abiertas. Curso de español para necesidades inmediatas I y su continuación Puertas Abiertas. Curso de español para necesidades inmediatas II, en adelante nos referiremos a ellos como Puertas Abiertas I y Puertas Abiertas II.

Estos cursos se albergan en la plataforma $O P E N e d X^{2}$ que, fundada en 2012 sin ánimo de lucro por la Universidad de Harvard y el Massachussets Institut of Technology (MIT), proporciona soporte técnico para acoger cursos procedentes de universidades e instituciones de todo el mundo.

Se trata de dos cursos diseñados y desarrollados por el grupo de investigación AT$\mathrm{LAS}^{3}$ de la Universidad Nacional de Educación a Distancia (España) dentro del marco del proyecto europeo MOONLITE ${ }^{4}$ (Massive Open Online courses eNhancing LInguistic and Transversal skills for social inclusion and Employability) que se llevó a cabo entre 2016 y 2019 (Read, Bárcena, Arús \& Sedano, 2017). Se ofrecen dentro del catálogo de formación del servicio de cursos gratuitos de UNED Abierta ${ }^{5}$ desde 2019. El público al que se dirigen son personas desplazadas en general con especial atención a migrantes o refugiadas.

En este sentido, los dos LMOOC Puertas Abiertas tienen como objetivo fomentar

2 OPENedX: https://open.edx.org/ [31/05/2021].

3 ATLAS (Applying Technology to Languages): http://portal.uned.es/portal/page?_pageid=93,8842771\&_ dad=portal\&_schema=PORTAL [31/05/2021].

4 Proyecto europeo Erasmus + MOONLITE, Key Action 203 - Strategic Partnerships for Higher Education, ref. 2016-1-ES01-KA203-02573.

5 UNED Abierta: https://blogs.uned.es/unedabierta/ [31/05/2021]. 
habilidades lingüísticas, interculturales, y transversales con vistas a facilitar la inclusión social y la empleabilidad de este colectivo, especialmente vulnerable tanto en el país de origen como en el de acogida, víctimas, en la mayoría de los casos, de situaciones de conflicto en sentido amplio (político, social o laboral). Porque, tal y como señalan Collier $\&$ Betts (2017), no es solo huir del conflicto lo que convierte a los refugiados en víctimas sino también negarles los medios para volverse autónomos y productivos, atendiendo únicamente a las necesidades básicas de alimentos y refugio cuando son acogidos. El acceso a la educación y al trabajo son piezas primordiales para facilitar la integración en el país de destino. Ya en 1951, la convención sobre el estatuto de derechos de los refugiados hacía especial énfasis en promover la autonomía y el acceso al trabajo como estrategias fundamentales de admisión (ACNUR, 1951). Los informes anuales de las instituciones responsables de la recepción (ACNUR, ONGs, etc.) concluyen, además, que, en la mayoría de los casos, esta condición de desplazados se prolonga en el tiempo por lo que se hace cada vez más necesario que este colectivo pueda tener acceso al sector productivo. En consecuencia, la formación en la lengua y cultura del país de acogida que asegure una comunicación efectiva entre la población nativa y la alófona, se revela como un instrumento clave para favorecer dicha integración.

Estos dos LMOOC responden a un exhaustivo análisis de necesidades formativas, vivenciales, vitales y tecnológicas realizado junto con 16 entidades de apoyo y recepción de personas migrantes y refugiadas que colaboraron con el proyecto (Castrillo \& Martín-Monje, 2018; Martín-Monje, Castrillo \& Sedano, 2019). Así pues, a nivel técnico se caracterizan por su usabilidad y su accesibilidad, es decir, no solo incorporan una guía de uso y soporte técnico, sino que también la navegación por el entorno es sencilla, pueden realizarse con una conexión inestable, permiten descargar todo tipo de materiales (vídeo, audio, imagen, PDF), los recursos audiovisuales son de resolución baja para facilitar dicha descarga, se adaptan a dispositivos móviles, contienen subtítulos, transcripciones y están adaptados para responder ante dificultades visuales (Arús, 2019).

En cuanto a las características lingüísticas e interculturales destacan por (1) la sencillez de expresión, utilizando un registro adaptado a los niveles de competencia lingüística, así como una velocidad razonable de habla en los vídeos y audios, (2) la atención al multilingüismo incluyendo un glosario de términos y subtítulos traducidos al inglés, francés y árabe, lenguas vehiculares identificadas como mayoritarias entre la población migrante, (3) la atención a la diversidad de identidades y procedencias en materiales y actividades y (4) el fomento de la conciencia y comunicación interculturales entre los participantes a través de las actividades que se proponen.

Finalmente, a nivel pedagógico, se caracterizan por ser masivos y abiertos en todos los sentidos (sin requisitos académicos o administrativos, gratuitos, incluidos los certificados de realización; los materiales y recursos disponibles al acabar el curso; tiene en cuenta las diferencias horarias en contexto internacional), están centrados en las necesidades de los 
participantes y son de carácter práctico. Se presentan en formato modular, flexible y progresivo, incluyen temas obligatorios para la obtención del certificado y opcionales, y ofrecen un andamiaje en todo momento con retroalimentación positiva por parte de los facilitadores.

En relación con los contenidos y la metodología de los dos cursos, estos están enfocados a (1) facilitar el aprendizaje del español en los aspectos básicos de la vida y los aspectos culturales que se derivan de las situaciones comunes a las que se tendrán que enfrentar, así como (2) a fomentar el aprendizaje autónomo y la práctica colaborativa del español oral y escrito en línea.

Están organizados para trabajarse durante 6 semanas, cada uno con 4 módulos de contenido, uno por semana, tras una primera destinada a familiarizarse con la plataforma y los materiales y una final de revisión o para completar tareas pendientes. Se estima una dedicación de tiempo de unas 25 horas por curso. Los temas propuestos ayudarán a los participantes a realizar trámites, desenvolverse por la ciudad, hacer planes, buscar vivienda, ir al médico o defender los derechos, entre otros. En este sentido, los títulos de los módulos adelantan parte de las cuestiones que se abordan:

\author{
Puertas Abiertas I \\ Módulo 1: ¡Hola! Aquí estamos \\ Módulo 2: Hacemos la vida diaria \\ Módulo 3: Nos movemos \\ Módulo 4: Vamos al médico \\ Puertas Abiertas II \\ Módulo 1: Hacemos planes \\ Módulo 2: Buscamos casa \\ Módulo 3: Estudiamos y trabajamos \\ Módulo 4: Defendemos nuestros derechos.
}

Cada módulo se compone de actividades de aplicación práctica en contextos de integración y empleabilidad, así como foros de debate en los que los participantes exponen sus experiencias y puntos de vista, explican aspectos cotidianos de su país y cultura de origen en contraste con la española, revelándose estos como verdaderos puntos de encuentro entre personas distantes en el espacio, en la lengua y en la cultura, pero con un interés común, el de la intercomprensión y el entendimiento.

\title{
3. LMOOC para el aprendizaje del Francés como Lengua Extranjera
}

Tal y como hemos avanzado en la introducción, los LMOOC para el aprendizaje de Francés como Lengua Extranjera que hemos seleccionado, están destinados a dos colectivos diferentes de los LMOOC Puertas Abiertas. En este caso, el público meta son las personas que deseen desplazarse a Francia por motivos profesionales o académicos. 
Desde hace poco más de una década, la imparable globalización en la que se encuentran todos los sectores, económico, político, sanitario educativo, social, etc. ha tenido como consecuencia que las instituciones de enseñanza superior incluyan en su hoja de ruta la internacionalización como prioridad estratégica, internacionalización que "no solo implica la movilidad de las personas, sino también la movilidad de cursos, programas e instituciones", como puntualizan López \& Almaraz (2020: 101).

La selección de cursos que hacemos en este apartado responde a una constatación, también expresada por López \& Almaraz (2020: 101): “en un mundo cada vez más globalizado los jóvenes estudian en el extranjero y los profesionales cualificados cruzan las fronteras en pos de oportunidades de empleo". En este sentido, los tres LMOOC de FLE tienen en común el objetivo de favorecer la inmersión de cualquier persona desplazada en contextos profesionales y/o educativos en el país de destino. Estos son:

- LMOOC Travailler en France A2-B1

- LMOOC Mooc2move: le français pour l'université

- $\quad$ LMOOC Étudier en France:French Intermediate course B1-B2.

A diferencia de la exposición de los dos anteriores, a continuación, describiremos cada uno de los tres LMOOC de manera independiente puesto que se proponen desde instituciones y plataformas diferentes y no tienen ningún tipo de vinculación entre sí.

\subsection{LMOOC Travailler en France A2-B1}

Se trata de un LMOOC diseñado por la Alliance Française Paris Île-de-France (Francia) y ofertado dentro del catálogo de la plataforma de educación virtual France Université Numérique (FUN MOOC) ${ }^{6}$, creada en 2013 y desarrollada por el Ministerio de Educación Nacional francés con el objetivo de ofrecer formación masiva de carácter virtual con cursos en francés y en inglés de temática variada. El proveedor de formato que alberga estos MOOC es, como en los dos anteriores, OPENedX.

El LMOOC Travailler en France A2-B1 se inició el 31 de marzo de 2019, organiza sesiones cada tres meses, pero puede realizarse a lo largo de todo el año, según figura en la página de bienvenida, y, estará disponible para recibir nuevas inscripciones hasta el 1 de enero de 2022, previendo su finalización el 1 de abril de 2022.

Su principal objetivo es ayudar a las personas que desean instalarse en Francia por motivos laborales, a través de la integración lingüística y sociocultural, acompañándolas en el aprendizaje, desde la búsqueda de un empleo o la preparación de una entrevista de trabajo hasta la incorporación en la empresa y la relación con los colegas.

En cuanto a las características técnicas, este LMOOC presenta un formato versátil

6 FUN MOOC: $<$ https://www.fun-mooc.fr/fr/> [31/05/2021]. 
adaptable para dispositivos móviles y de navegación sencilla e intuitiva, lo que garantiza la usabilidad.

En el plano lingüístico e intercultural se parte de un nivel de competencia lingüística de $\mathrm{A} 2^{7}$, se imparte exclusivamente en francés, pero incluye la transcripción de los audios y de los vídeos para facilitar la comprensión de los contenidos, si fuera preciso. También ofrece la posibilidad de regular la velocidad de habla de estos para adaptarse al grado de competencia de los participantes. No proporciona ninguna certificación oficial de dominio de lengua, pero los participantes pueden recibir una credencial de seguimiento del curso habiendo completado y superado, al menos, el $50 \%$ de las actividades propuestas. Los aspectos culturales e interculturales que aborda están relacionados con el ámbito profesional.

A nivel pedagógico hay que señalar que contiene una organización flexible, sin orden preestablecido, que permite a los participantes la libre elección de actividades y temas. A diferencia del resto de LMOOC que describimos, en este, el equipo pedagógico no menciona ninguna estimación de horas de dedicación al curso, solo se alude a ediciones de tres meses para poder obtener el certificado de seguimiento.

Los contenidos tratados focalizan la atención en las particularidades del contexto y legislación laboral franceses y se organizan en torno a dos bloques: (1) uno general que ofrece herramientas transversales comunes para todo tipo de empleos sobre: cómo buscar un empleo, contestar a una oferta de empleo, preparar una entrevista de trabajo, integrarse en una empresa, trabajar en equipo y comunicar con otros colegas, y (2) otro específico que invita a descubrir diferentes profesiones ligadas a los principales sectores de reclutamiento de trabajadores, a saber: la construcción, la hostelería y la restauración, la informática, la salud y los servicios de apoyo a personas y empresas

Listamos a continuación los temas propuestos en el bloque general cuyos títulos, expresados en primera persona, Je, facilitan la identificación del usuario con la propuesta formativa. Estos son:

- Je recherche un emploi
- Je m'intègre dans l'entreprise
- Je participe à la vie sociale
- Je gère des situations inhabituelles
- Je fais un bilan et je développe mes compétences
- Je travaille en collaboration.

parte, los títulos del bloque específico hacen referencia a los sectores mencionados anteriormente y despliegan como subtítulos el nombre de las profesiones, que indicamos entre paréntesis, abordadas en los contenidos, facilitando igualmente el reconocimiento de los usuarios con posibles puestos de trabajo a los que podrían optar, a saber:

7 A lo largo de este trabajo haremos referencia a diferentes niveles de competencia lingüística (A1, A2, B1, B2) según la escala establecida por el Conseil de l'Europe (2001) en el Cadre européen commun de référence pour les langues. 
- Bâtiment (Chef de chantier, Maçon, Chargée d'affaires)

- Hôtellerie/restauration (Réceptionniste, Gouvernante, Employée polivalente de restautarion)

- Informatique (Développeuse web, Technitioen Hot Line, Responsable de sécurité)

- Santé (Médecin, Infirmière, Aide-soignante)

- Services aux personnes et aux entreprises (Auxiliaire de vie sociale, Agent d'entretien, Magasinier).

Finalmente, este LMOOC también propone foros de discusión en cada uno de los temas en los que los participantes expresan, sobre todo, sus dudas y plantean preguntas relacionadas con la temática correspondiente, en lugar de establecer debate propiamente dicho, reflejo sin duda de la inquietud que provoca tanto el hecho de desplazarse a otro país, como el de acceder a un puesto de trabajo. Así pues, encontramos hilos de conversación referidos a consultas sobre aspectos prácticos y concretos del ámbito profesional, dudas sobre trámites legales, la traducción de diplomas o cargos para un Curriculum Vitae, el calendario laboral, las competencias más solicitadas o la homologación de títulos, por citar algunos. Es importante señalar que estos foros están abiertos en el tiempo y no siempre cuentan con personal pedagógico responsable de responder, por lo que, prácticamente, son los propios participantes quienes resuelven las dudas y comentan las intervenciones del resto de compañeros, creando así una verdadera comunidad de aprendizaje virtual.

\subsection{LMOOC Mooc2move: le français pour l'université}

Mooc2move: le français pour l'université se encuentra, igualmente, en el catálogo de MOOC que brinda la plataforma virtual FUN MOOC y, por lo tanto, con el formato OPEedX. La institución que lo ha diseñado y desarrollado es la Université de Grenoble Alpes (Francia). La edición que se toma como referencia para nuestro estudio se ha desarrollado entre el 17 de abril de 2021 y el 24 de mayo de 2021.

El objetivo principal de este LMOOC es facilitar la inmersión de los estudiantes internacionales en el sistema universitario francés y prepararlos para estudiar en Francia, ofreciéndoles formación lingüística y cultural.

En cuanto a las características técnicas, también dispone de un formato versátil y sensible, adaptable a dispositivos móviles, aunque algunas pruebas evaluables solo pueden realizarse por ordenador. Cuenta con un módulo 0 que ayuda a los usuarios a familiarizarse con el entorno virtual, contiene varios tutoriales que explican cada uno de los elementos que aparecen en la interfaz e incluye un foro para resolver las cuestiones técnicas.

A nivel lingüístico e intercultural, parte de un nivel de competencia lingüística de B1, tanto la lengua vehicular como la de instrucción es el francés, pero facilita la transcripción de los vídeos y audios con el fin de facilitar la compresión, cuando sea necesario, a través del soporte escrito. Del mismo modo, la velocidad de habla en ellos es regulable por los propios 
usuarios. No proporciona certificación oficial del dominio de lengua, pero emite una credencial de finalización del curso tras la superación mínima del 50\% de las pruebas. Relacionado con los contenidos, se trabajan los registros de lengua formal e informal y se dan consejos para comunicar con éxito tanto oralmente como por correo electrónico, por ejemplo.

Respecto a las características pedagógicas, se organiza en 6 módulos independientes, que se activan semana a semana, compuestos por vídeos cortos y diversas actividades. Los vídeos utilizan testimonios de alumnos, personal administrativo y profesores que explican ciertas especificidades académicas francesas. Propone actividades de evaluación por pares que, combinadas con el resultado de cuatro pruebas finales, permitirán obtener un certificado de finalización con éxito. Se estima una dedicación de tiempo total de 24 horas.

Los contenidos de este LMOOC, centrados en las particularidades del sistema educativo francés, están encaminados a guiar a los futuros estudiantes internacionales por diferentes escenarios de su vida académica, desde las primeras gestiones que deben realizar antes de su llegada a Francia, pasando por cuestiones cotidianas relacionadas con el alojamiento, salud, trámites bancarios, hasta aspectos más académicos como la matrícula en la universidad o procedimentales como son la toma de notas, la interpretación de datos, la citación de referencias o la preparación de una exposición oral, los típicos exposés. Los títulos de los seis módulos anuncian estos contenidos:

- Módulo 1: Étudier en France

- Módulo 2: Bien communiquer: avec qui, comment, pourquoi

- Módulo 3: Comprendre ses cours à l'université

- Módulo 4: Interpréter des données chiffrées

- Módulo 5: Se documenter et citer

- Módulo 6: Faire un exposé.

Finalmente, como todos los LMOOC, aprovecha el recurso del foro de debate para incitar a los participantes a intervenir y compartir experiencias, inquietudes o solicitar ayuda o consejo. Así pues, observamos que aparecen temas tan diversos y ricos como son las representaciones que los participantes tienen sobre Francia y/o la lengua y/o cultura francesas; reflexiones sobre de la pragmática de los diferentes registros de lengua comparando el francés con otras lenguas como el italiano o el japonés; opiniones acerca de los clichés sobre las diferentes lenguas (béret rouge et baguette, por ejemplo, en el caso de Francia); explicaciones sobre los tipos de clases convencionales en el sistema universitario francés y la habitual manera de nombrarlos por sus siglas (CM, TD, $\mathrm{TP}^{8}$, etc.); argot y expresiones propias del habla de los jóvenes, como el verlan o la abreviación de palabras, por ejemplo, o los dialectos que se hablan en los países de origen.

8 CM: Cours Magistraux; TD: Travaux Dirigés; TP: Travaux Pratiques. 


\subsection{LMOOC Étudier en France: French Intermediate B1-B2}

Se trata de un curso diseñado por l'École Polytechnique de Paris (Francia), está alojado y figura dentro del catálogo de cursos de la plataforma Coursera ${ }^{9}$, una de las primeras y más importantes plataformas proveedoras de cursos MOOC. Fundada en 2011 por los profesores de la Universidad de Standford, Daphne Koller y Andrew Ng, Coursera se crea con el objetivo de ofrecer formación y aprendizaje para cualquier persona y en cualquier rincón del planeta. Actualmente es líder en educación superior que da formación a más de 77 millones de usuarios, según se indica en su página web, y más de 200 universidades, empresas punteras y educadores de todo el mundo proponen cursos de especialización o diplomas.

Este curso de francés de nivel intermedio B1/B2, forma parte de un sistema dual de "formación con tecnología digital" y "lengua e interculturalidad" puesto en marcha por la Université Paris Saclay, institución que respalda este LMOOC. Está dirigido a todos los estudiantes que deseen realizar sus estudios superiores en Francia y tiene como objetivo no solo prepararlos para obtener la competencia de lengua necesaria para seguir las clases con garantía en cualquier universidad francesa en términos de competencias lingüísticas, sino también ayudarles en la adaptación a su nuevo entorno educativo y social en las mejores condiciones.

Del mismo modo que los cursos anteriormente descritos, técnicamente este LMOOC también presenta un formato versátil, adaptable a los dispositivos móviles, de navegación sencilla e interfaz clara y amable. Incorpora un centro de ayuda técnica identificado con un signo de interrogación en el que se pueden formular preguntas o escribir palabras clave relacionadas con cualquier cuestión técnica o de funcionamiento, el sistema devuelve una batería de artículos con información relacionada con lo solicitado, similar a las conocidas preguntas frecuentes $\left(\mathrm{FAQ}^{10}\right)$ que suele haber en las páginas web.

Respecto a las características lingüísticas e interculturales, el nivel de lengua de partida es B1 y se ofrece exclusivamente en francés "pour aider à progresser", según la descripción del curso, aunque incluye también la transcripción de los audios y de los vídeos, así como algunas fichas de vocabulario o de ampliación de materia que facilitan la realización de los ejercicios. También permite regular la velocidad de habla en los documentos orales. No proporciona certificación oficial de competencia lingüística conseguido y así lo menciona explícitamente "Note importante: ce Mooc ne remplace pas la passation de tests attestant du niveau B1 ou B2 tels que ceux proposés par le Ciep: TCF, Delf ou Dalf', pero se puede obtener gratuitamente una credencial de finalización del curso habiendo superado el $50 \%$ de las actividades.

Lo que más llama la atención a nivel pedagógico es la flexibilidad y la adaptación del programa a los ritmos de trabajo de los participantes. En efecto, se plantea con una duración de 6 semanas, en las que se organizan los contenidos y las actividades, pero se encuentra dis-

9 Coursera: <https://www.coursera.org/>[31/05/2021].

10 Las siglas FAQ pueden responder a Frequently Asked Questions, en inglés, o a Foire Aux Questions, en francés. 
ponible para poder iniciarlo en cualquier momento. Por otra parte, los participantes, una vez inscritos, tienen la posibilidad de reestablecer sus fechas límite de realización y envío de las actividades, en caso de no poder realizarse de manera continuada; del mismo modo se puede ajustar su huso horario, haciendo de esta, una formación flexible y personalizada adaptada a los usuarios. Se estima un tiempo de trabajo de 46 horas. Otro elemento que facilita la personalización del aprendizaje es que deja a los aprendices definir el tiempo y los días que prevén dedicar a la realización del curso. Inconscientemente, esto crea un compromiso personal con el propio aprendizaje y favorece el mantenimiento de un ritmo más regular.

Los contenidos de este curso están diseñados para facilitar la inmersión de los participantes en el mundo estudiantil a través de textos en múltiples formatos, extractos de películas y vídeos auténticos que dan voz a estudiantes franceses o extranjeros que viven en Francia. Como se puede observar a continuación, los títulos que recoge cada propuesta formativa semanal dan idea de la progresión temática seguida, desde la llegada al país hasta la plena integración social:

\section{- Semana 1: Arriver en France \\ - Semana 2: S'adapter à la vie en France \\ - Semana 3: Devenir étudiant en France \\ - Semana 4: Assister à un cours \\ - Semana 5: Communiquer et s'intégrer \\ - Semana 6: Vivre en France.}

En cada tema, además de los documentos en vídeo y texto que los introducen, se incluyen diferentes tipos de actividades que permiten trabajar todas las destrezas: comprensión oral y escrita, expresión oral y escrita, gramática y vocabulario.

Las actividades de cada semana se acompañan de un foro de discusión relacionado con la temática abordada en el momento: clichés y prejuicios; opinión sobre la apertura de establecimientos comerciales en domingo; opinión sobre estudios superiores privados; sistemas de evaluación en la universidad; derechos del Hombre y del ciudadano; a propósito de la felicidad.

Los responsables del curso abren los foros con mensajes que recogen la temática de la semana, apelan a algún documento o actividad realizada y solicitan la opinión de los participantes, en la mayoría de los casos, preguntando por cómo se trata ese asunto en su país, tal y como podemos ver en la siguiente cita, extraída del foro de la semana 2:

Dans votre vie quotidienne en France, vous irez faire des courses et parfois.... les magasins seront fermés. Pourquoi? Parce que la plupart des magasins ne sont ouverts ni la nuit ni le dimanche.

Que pensez-vous du dessin de Plantu?

Êtes-vous pour ou contre l〉ouverture des magasins le dimanche? Quel est le point de vue des Français sur ce sujet? Quels sont les avantages pour les employés? Pour les clients? Quels sont les inconvénients?

Dans votre propre pays, les magasins sont-ils ouverts le dimanche? 
Pensamos que esta manera de dirigirse a los aprendices, apelando a sus conocimientos $\mathrm{y}$, sobre todo, a sus vivencias particulares, a asuntos que les atañen, incita a la participación espontánea de los usuarios y hace que se cuenten por de miles los mensajes que registran estos foros, cada uno aportando su punto de vista personal y acercando su propia cultura y costumbres a los demás.

Convenimos con Casallas (2021) que es preciso entender la internacionalización de la educación como un proceso dinámico e integrador:

Es un proceso dinámico porque sus prácticas no son estáticas, sino que se renuevan a medida que cambian y se transforman las sociedades, así como a partir de los nuevos procesos y oportunidades que surgen con el desarrollo de las Tecnologías de la Información y la Comunicación (TIC). Por otro lado, es integrador en la medida en que genera vínculos entre los individuos, las instituciones, los Estados y las culturas. (Casallas, 2021: 60)

Una muestra de ello son los cursos que hemos expuesto, especialmente los dos últimos, creados desde instituciones universitarias para favorecer la internacionalización a través de la movilidad estudiantil. Cursos dinámicos, implementados mediante las TIC que generan vínculos entre individuos y culturas.

\section{Conclusión}

El auge de las tecnologías y en concreto de los dispositivos móviles, el uso exponencialmente masivo de las redes sociales, así como el aumento de contenidos de Internet en abierto, fácilmente accesibles, están dibujando un nuevo paisaje educativo que conviene no desdeñar, puesto que, tal y como hemos mostrado en las páginas precedentes, en muchos casos se trata de propuestas diseñadas por expertos e instituciones de avalado prestigio, que acreditan su calidad. Los 5 LMOOC que hemos presentado son una muestra de ello, a la vez que dan respuesta, a uno de los objetivos principales de los MOOC como es la democratización de la formación y el conocimiento facilitando el acceso a un vasto público por su carácter gratuito y exigencias tecnológicas mínimas.

Hemos podido constatar, igualmente, que, en los planteamientos pedagógicos de estas propuestas formativas, subyacen los dos principios que apuntaba Matilde Anquetil (2006) para desarrollar la competencia intercultural: diseñar actividades que obliguen al aprendiz a interactuar con el medio y actividades que propicien la creación de situaciones de encuentro. En esta línea de ideas, es importante mencionar que los MOOC, por su propia naturaleza y carácter masivo, se muestran como entornos idóneos que favorecen en sí mismos el encuentro intercultural, independientemente de la temática que se trate en ellos. En particular, hay que resaltar en ellos el papel que juegan los foros de discusión, espacios en los que se desdibujan las distancias, físicas y culturales, revelándose como un componente clave en el 
Anales de Filología Francesa, n. ${ }^{\circ}$ 29, 2021

LMOOC PARA LA INTEGRACIÓN DE PERSONAS DESPLAZADAS

encuentro intercultural, y en el caso de en los cursos descritos, propiciado, adicionalmente, por el tono cordial y el enfoque personalizado con los que se han planteado.

Los LMOOC, además, permiten integrar de manera orgánica en sus programaciones, contenidos tanto de corte lingüística como meramente intercultural.

Para terminar, constatamos que, estos cursos, por el hecho de poder realizarse desde la distancia, en el país de origen, en cualquier sitio, o en contexto de inmersión a la llegada al país de destino, se evidencian como excelentes herramientas de preparación previa, en el primer caso, y en el segundo, de acompañamiento lingüístico-cultural de todas las personas desplazadas, cualquiera que sea la razón que las ha llevado a ello, durante la estancia en el país de acogida.

\section{Referencias bibliográficas}

ACNUR. 1951. Convention and protocol relating to the status of refugees: $<\mathrm{https}: / / \mathrm{www}$. unhcr.org/uk/1951-refugee-convention.html> [31/05/2021].

ACNUR. 2020. Tendencias globales de desplazamiento forzado en 2019: <https://www. acnur.org/5eeaf5664.pdf> [31/05/2021].

Alliance FranÇaise PARIS-ÎLe-De-France. 2019. LMOOC Travailler en France A2-B1: $<$ https://www.fun-mooc.fr/fr/cours/travailler-en-france-a2-b1/> [31/05/2021].

Anquetil, Mathilde. 2006. Mobilité Erasmus et communication interculturelle. Berna, Peter Lang.

ARÚs HitA, Jorge. 2019. "Puertas Abiertas: MOOC de lenguas adaptado a las necesidades de migrantes y refugiados" in $V$ Jornadas ELE: las TIC en la enseñanza de ELE, Universidad Complutense de Madrid, 19-20 marzo 2019.

Bárcena Madera, Elena \& Elena Martín-Monje. 2014. "Introduction. Language MOOCs: An emerging field" in Martín-Monje, Elena \& Elena Bárcena Madera (Eds.). Language MOOCs: Providing learning, transcending boundaries. Berlin, De Gruyter, 1-15.

Casallas Herrera, Miguel Ángel. 2021. "Internacionalización de la Educación Superior: más allá de la movilidad académica" in Guerrero Escobar, Javier Iván (comp.) Internacionalización del currículo y experiencias pedagógicas. Pertinencia y aprendizaje global en educación superior. Bogotá, UNIMINUTO, 57-89.

Castrillo, María Dolores \& Elena Martín-Monje. 2018. "Integración Lingüística de Refugiados e Inmigrantes a Través de Los MOOC" in XXXVI CONGRESO AESLA Lingüística aplicada y transferencia del conocimiento: empleabilidad, internacionalización y retos sociales: <https://cutt.ly/nnRzpit> [31/05/2021].

Collier, Paul \& Alexander BetTs. 2017. "Why denying refugees the right to work is a catastrophic error" in The Guardian: <https://cutt.ly/ynRzh3r> [31/05/2021].

COMISIÓN EUROPEA. 2020. Erasmus + annual report 2019: <https://ec.europa.eu/programmes/erasmus-plus/about/statistics_es> [31/05/2021]. 
Anales de Filología Francesa, n. ${ }^{\circ}$ 29, 2021

MERCEDES SANZ GIL

CONSEIL DE L'Europe. 2001. Cadre européen commun de référence pour les langues. Paris, Les Éditions Didier.

École Polytechnique de Paris. 2021. LMOOC Étudier en France: French Intermediate B1-B2: <https://fr.coursera.org/learn/etudier-en-france> [31/05/2021].

ILostat. 2018. ILO Global Estimates on International Migrant Workers. Results and Methodology: <https://cutt.ly/8nRzzLi> [31/05/2021].

López Esteban, Carmen \& Fernando E. Almaraz Menéndez. 2020. “Migración, desplazamiento y educación universitaria: el reconocimiento de competencias y cualificaciones del profesorado" in Aulas Innovadoras en la Formación de los Futuros Educadores de Educación Secundaria. Salamanca, Ediciones Universidad Salamanca, 95-113.

Martín-Monje, Elena, María Dolores Castrillo \& Beatriz Sedano Cuevas. 2019. "Language MOOC Design for Social Inclusion through the Lens of Maker Culture" in CALICO Conference 2019. <https://cutt.ly/knULHEM> [31/05/2021].

Organización de las Naciones Unidas. 2015. Objetivos de Desarrollo Sostenible: $<$ https://cutt.ly/qnULVr8> [31/05/2021].

Pernías Peco, Pedro \& Sergio Luján Mora. (2013). "Los MOOC: orígenes, historia y tipos" in Centro de Comunicación y Pedagogía: <http://www.centrocp.com/los-mooc-origenes-historia-y-tipos/> [31/05/2021].

Read, Timothy, Bárcena Madera, Elena, ARÚS, Jorge y Beatriz Sedano Cuevas. 2017. "The Potential of Language MOOCs for the Social Inclusion of Displaced People", Conference Language Skills for Social and Economic Inclusion. Berlín.

SANZ GIL, Mercedes. 2020. "MOOC para comprender la interculturalidad de lo cotidiano" in Baynat Monreal, Ma Elena, Mercedes Eurrutia Cavero \& Catherine Sablé (eds.). TIC e Interculturalidad. Miradas Cruzadas. Granada, Editorial Comares (col. Interlingua), 323-345.

Sedano Cuevas, Beatriz. 2017. "Español para Viajeros”: Diseño de un MOOC de Lenguas a partir del análisis de necesidades formativas en el dominio turístico. Tesis doctoral, Universidad Nacional de Educación a Distancia.

SiEmens, Georges. 2004. "Conectivismo: Una teoría de aprendizaje para la era digital": $<$ https://cutt.ly/nuLOvUH $>$ [31/05/2021].

Universidad Nacional de Educación a Distancia. 2021. LMOOC Puertas abiertas: curso de español para necesidades inmediatas I: <https://iedra.uned.es/courses/coursev1:UNED+PuertasAbiertasI+2021/about> [31/05/2021].

Universidad Nacional de Educación a Distancia. 2021. LMOOC Puertas abiertas: curso de español para necesidades inmediatas II: <https://iedra.uned.es/courses/coursev1:UNED+PuertasAbiertasII+2021/about> [31/05/2021].

Université de Grenoble AlPes. 2021. LMOOC Mooc2Move: le français pour l'Université: $<$ https://www.fun-mooc.fr/fr/cours/mooc2move/> [31/05/2021]. 
